\title{
Sustaining Gains in Diabetic Eye Screening: Outcomes from a Stakeholder-Based Implementation Program for Teleophthalmology in Primary Care
}

Yao Liu, MD, MS, ${ }^{1}$ Julia N. Carlson, BS, ${ }^{1}$

Alejandra Torres Diaz, BS, ${ }^{1}$ Loren J. Lock, MS, ${ }^{1}$

Nicholas J. Zupan, $M P H^{1}{ }^{1}$ Todd D. Molfenter, $P h D^{2}$

Jane E. Mahoney, MD, ${ }^{3}$ Mari Palta, PhD, ${ }^{4,5}$ Deanne Boss, MS, ${ }^{6}$

Timothy D. Bjelland, DO, and Maureen A. Smith, PhD ${ }^{4,6}$

${ }^{1}$ Department of Ophthalmology and Visual Sciences, University of Wisconsin School of Medicine and Public Health, Madison,

Wisconsin, USA.

${ }^{2}$ Department of Industrial and Systems Engineering, University of Wisconsin-Madison, Madison, Wisconsin, USA.

Departments of ${ }^{3}$ Medicine, ${ }^{4}$ Population Health Sciences,

${ }^{5}$ Biostatistics and Medical Informatics, and ${ }^{6}$ Family Medicine and Community Health, University of Wisconsin School of Medicine and Public Health, Madison, Wisconsin, USA.

${ }^{7}$ Mile Bluff Medical Center, Mauston, Wisconsin, USA.

This study was presented at the 2019 Association for Research in Vision and Ophthalmology (ARVO) Annual Meeting in Vancouver, British Columbia, Canada, the 12th Annual Conference on the Science of Dissemination and Implementation in Health in Arlington, Virginia, and the 2020 American Telemedicine Association Telehealth Virtual Conference.

\section{Abstract}

Background: Teleophthalmology is a validated method for diabetic eye screening that is underutilized in U.S. primary care clinics. Even when made available to patients, its long-term effectiveness for increasing screening rates is often limited.

Introduction: We hypothesized that a stakeholder-based implementation program could increase teleophthalmology use and sustain improvements in diabetic eye screening.

Materials and Methods: We used the NIATx Model to test a stakeholder-based teleophthalmology implementation program, I-SITE at one primary care clinic (Main) and compared teleophthalmology use and diabetic eye screening rates with those of other primary care clinics (Outreach) within a U.S. multipayer health system where teleophthalmology was underutilized.
Results: Teleophthalmology use increased post-I-SITE implementation (odds ratio $[O R]=5.73$ [p $<0.001]$ ), and was greater at the Main than at the Outreach clinics (OR=10.0 vs. $1.69, \mathrm{p}<0.001)$. Overall diabetic eye screening rates maintained an increase from $47.4 \%$ at baseline to $60.2 \%$ and $64.1 \%$ at 1 and 2 years post-I-SITE implementation, respectively $(\mathrm{p}<0.001)$. Patients who were younger $(O R=0.98$ per year of age, $\mathrm{p}=0.02)$ and men $(O R=1.98, \mathrm{p}=0.002)$ were more likely to use teleophthalmology than in-person dilated eye examinations for diabetic eye screening.

Discussion: Our stakeholder-based implementation program achieved a significant increase in overall teleophthalmology use and maintained increased post-teleophthalmology diabetic eye screening rates.

Conclusion: Stakeholder-based implementation may increase the long-term reach and effectiveness of teleophthalmology to reduce vision loss from diabetes. Our approach may improve integration of telehealth interventions into primary care.

Keyword: ophthalmology, technology, telemedicine, telehealth

\section{Introduction}

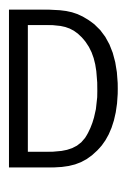

iabetic eye disease is the leading cause of blindness among working-age adults in the United States, primarily because of lack of access to eye screening. ${ }^{1}$ Approximately half of the 34 million U.S. adults with diabetes are adherent with American Diabetes Association (ADA) guidelines $^{2}$ for diabetic eye screening. ${ }^{3,4}$ Teleophthalmology is a validated form of diabetic eye screening shown to prevent blindness. ${ }^{5,6}$ This technology allows patients to obtain diabetic eye screening by having their eyes photographed using retinal cameras located in the primary care clinic where $90 \%$ of patients with diabetes regularly obtain care. ${ }^{7}$ Teleophthalmology is more convenient and accessible for patients than traditional screening, which requires a separately-scheduled, in-person dilated eye examination with an eye care provider.

(C) Yao Liu et al., 2020; Published by Mary Ann Liebert, Inc. This Open Access article is distributed under the terms of the Creative Commons Attribution Noncommercial License [CC-BY-NC] (http://creativecommons.org/licenses/by-nc/4.0/) which permits any noncommercial use, distribution, and reproduction in any medium, provided the original author(s) and the source are cited. 


\section{LIU ET AL.}

Unfortunately, teleophthalmology is significantly underutilized in U.S. primary care clinics. ${ }^{8,9}$ The 2020 Centers for Medicare and Medicaid Services (CMS) response to the COVID19 pandemic ${ }^{10}$ has improved telehealth reimbursement and expanded access to much-needed specialty care. However, challenges to its use remain even when teleophthalmology is made freely available to patients. A recent 5-year randomized controlled trial by Mansberger et al. ${ }^{11}$ found a promising initial increase in diabetic eye screening rates using teleophthalmology in primary care clinics. However, screening rates declined within 18 months and remained at $55 \%$ or lower thereafter despite teleophthalmology being offered to patients at no cost.

Successful teleophthalmology programs in the United States have been largely limited to a few settings. These include health systems where insurance coverage requires patients to obtain primary care and eye care within the same health system (Veteran's Administration [VA], Kaiser Permanente, etc.), ${ }^{3,12}$ large county health systems, ${ }^{13}$ or researchfunded programs. ${ }^{14}$ These settings have unique financial structures not representative of multipayer U.S. health systems where the majority of patients receive care. ${ }^{15}$

Our study was conducted at the Mile Bluff Medical Center, a U.S. multipayer health system where a teleophthalmology program had been established, but was very underutilized. Our earlier qualitative work and that of others have identified major workflow-related barriers to teleophthalmology use. ${ }^{8,16,17}$ To overcome those barriers, we hypothesized that a stakeholder-based implementation program could increase teleophthalmology use and diabetic eye screening rates through improved integration of teleophthalmology into primary care workflows.

In this study, we used the NIATx Model, ${ }^{18}$ a systematic health care process improvement framework, to engage patients and clinical stakeholders (i.e., primary care providers [PCPs], patient care staff, and administrators). We tested whether our stakeholder-based implementation program could sustain increased teleophthalmology use and diabetic eye screening rates.

\section{Materials and Methods}

\section{RESEARCH SETTING}

Mile Bluff Medical Center is a rural, U.S. multipayer health system located in Juneau County, WI. Juneau County ranks in the lowest quartile of Wisconsin counties based on health and socioeconomic factors. ${ }^{19}$ In 2015, a teleophthalmology program was developed based on the 2011 American Telemedicine Association Telehealth Practice Recommendations for Diabetic Retinopathy. ${ }^{20}$ A detailed description of the program has been previously published. ${ }^{16}$ After the teleophthalmology program was established, it was very underutilized (averaging $<20$ patients imaged per quarter).

\section{STUDY DESIGN}

Our earlier work on barriers and facilitators to teleophthalmology use suggested that a stakeholder-based implementation program focused on overcoming primary care workflow barriers may increase teleophthalmology use and increase diabetic eye screening. ${ }^{16}$ The implementation program allows for continuous modification of teleophthalmology implementation strategies by clinical stakeholders with patient input as described below. The Main clinic received the implementation program, whereas the remaining four Outreach clinics were waitlisted and serve as a comparator group for the present analyses. This study reports outcomes from testing the implementation program at the Main clinic.

\section{PATIENT AND CLINICAL STAKEHOLDERS}

We recruited Mile Bluff patients and clinical stakeholders in March 2017, 2 years after the teleophthalmology program was established, to participate in two separate stakeholder groups. We recruited adult patient stakeholders with diabetes who had previous teleophthalmology imaging or expressed interest in participating in research when contacted in a prior diabetic eye screening survey ${ }^{16}$ Patients were mailed an invitation letter from the Mile Bluff Chief Medical Officer, which included an opportunity to opt-out, followed by a phone call from our research team.

For the clinical stakeholders, we recruited PCPs and patient care staff during a presentation at a regularly scheduled Mile Bluff staff meeting. Additional clinical stakeholders included clinic administrators, quality improvement, and information technology/electronic health record staff who were selected for participation by Mile Bluff leadership to facilitate the design and testing of implementation strategies.

\section{I-SITE IMPLEMENTATION PROGRAM}

Our implementation program, I-SITE (Implementation to Sustain Impact for Teleophthalmology), applies the NIATx Model, ${ }^{18}$ a systematic health care process improvement framework. The NIATx Model outlines the roles of powerful leaders in the health system in creating system-level change. It also specifies the processes necessary to engage these leaders and access the personnel and resources necessary to streamline telehealth workflows. We used this framework to guide our clinical stakeholder meetings and iterative testing of implementation strategies for increasing teleophthalmology use at the Main clinic.

An experienced practice facilitator (D.B.) led the clinical stakeholder group through a series of three meetings over 4 
weeks to identify the major barriers to teleophthalmology use and strategies to overcome those barriers. Iterative testing of the selected implementation strategies then began at the Mile Bluff Main clinic. We then brought together a subset of the clinical stakeholders, including PCPs and patient care staff who practiced solely at the Main clinic, to form a local implementation team. This team met for 30 min monthly to review data on teleophthalmology use and clinical staff feedback. Based on this information, the team made decisions regarding whether to continue, abandon, or further refine each implementation strategy, as well as to propose new implementation strategies.

A similar process was used to solicit patient stakeholder input on major patient barriers and strategies to increase teleophthalmology use and diabetic eye screening. Thus, our implementation program allowed key stakeholders to continuously tailor and refine implementation strategies to address the unique needs and resources of the clinic and the patient population. Some strategies tested included audit and feedback reports, ${ }^{21}$ provider performance-based incentives, revised patient education materials, and sending patient reminders.

\section{TELEPHONE SURVEY AND MEDICAL RECORDS REVIEW}

Diabetic eye screening rates at all clinics were assessed using a patient telephone survey and medical records review conducted over 2-3 weeks in each year from 2015 to 2019. We used this method because Mile Bluff does not have complete diabetic eye screening records from eye care providers practicing outside their health system, a common situation in multipayer health systems. ${ }^{16,22}$

Patients were selected for survey participation using a random-number generator in Microsoft ${ }^{\circledR}$ Excel $^{\mathrm{TM}}$ (Microsoft Corp., Redmond, WA). They were sent a letter inviting their participation from the Mile Bluff Chief Medical Officer, with an option to opt-out if they preferred not to be contacted. Minimum survey sample sizes were calculated to achieve a 95\% confidence interval $( \pm 10 \%)$ in the diabetic eye screening rate at each clinic. The 2018 diabetic eye screening rate survey, for which we had complete medical record abstraction data, was performed over 3 weeks from August to September 2018.

Patients were considered unable to be contacted by phone after at least six attempts at different times of the day. Medical records from the Mile Bluff electronic health record and those from the patient's self-reported eye care provider were reviewed to confirm the date and type of their most recent diabetic eye screening (i.e., in-person dilated eye examination or teleophthalmology). For those patients who selfreported diabetic eye screening within the preceding 12 months, but we were unable to obtain confirmatory records from the patient's eye care provider (e.g., provider's office had closed with no access to records), we imputed adherence based on the proportion of patients with available medical records that had accurately self-reported screening, because patient self-report of diabetic eye screening is known to overestimate adherence. ${ }^{23}$

\section{OUTCOME MEASURES}

The primary outcome measures were teleophthalmology use and diabetic eye screening rates at the Main and Outreach clinics at 1 and 2 years post-I-SITE implementation. Teleophthalmology use was defined as the number of patients who obtained teleophthalmology imaging based on medical records review. Diabetic eye screening was defined as a medical record-confirmed dilated eye examination with an eye care provider or teleophthalmology imaging within the past 12 months following guidelines from the American Academy of Ophthalmology (AA0). ${ }^{24}$ Eligible patients had a diagnosis of diabetes mellitus and had seen a PCP located at the Main clinic $(n=989)$ or an Outreach clinic $(n=665)$ within the past 2 years.

\section{STATISTICAL ANALYSES}

Our primary data analysis used Mantel-Haenszel chisquared analysis to compare the odds of teleophthalmology use and diabetic eye screening between the Main and Outreach clinics across three time periods: baseline (March 2014-April 2015), post-teleophthalmology (April 2015-April 2017), and post-I-SITE implementation at the Main clinic (April 2017October 2018). We subsequently obtained further data on teleophthalmology use and diabetic eye screening rates extending through quarter 2 of 2019.

We used $t$-tests and chi-squared tests to assess for demographic differences between diabetic eye screening survey participants and nonparticipants using data from the 2018 diabetic eye screening rate survey. We used multivariable logistic regression on the same data set to identify associations of age, gender, hemoglobin A1c levels, and clinic location with diabetic eye screening in the preceding 12 months ad with the type of screening obtained (i.e., in-person dilated eye examination or teleophthalmology). All statistical analyses were performed using Microsoft Excel (Microsoft Corp.) and the R statistical software program (R Foundation for Statistical Computing, Vienna, Austria).

\section{ETHICS AND INSTITUTIONAL REVIEW BOARD REVIEW}

This study was reviewed by the UW Human Subjects Institutional Review Board (IRB) staff and was determined to be exempt from full IRB review because the activities were consistent with providing evidence-based quality improvement 


\section{LIU ET AL.}

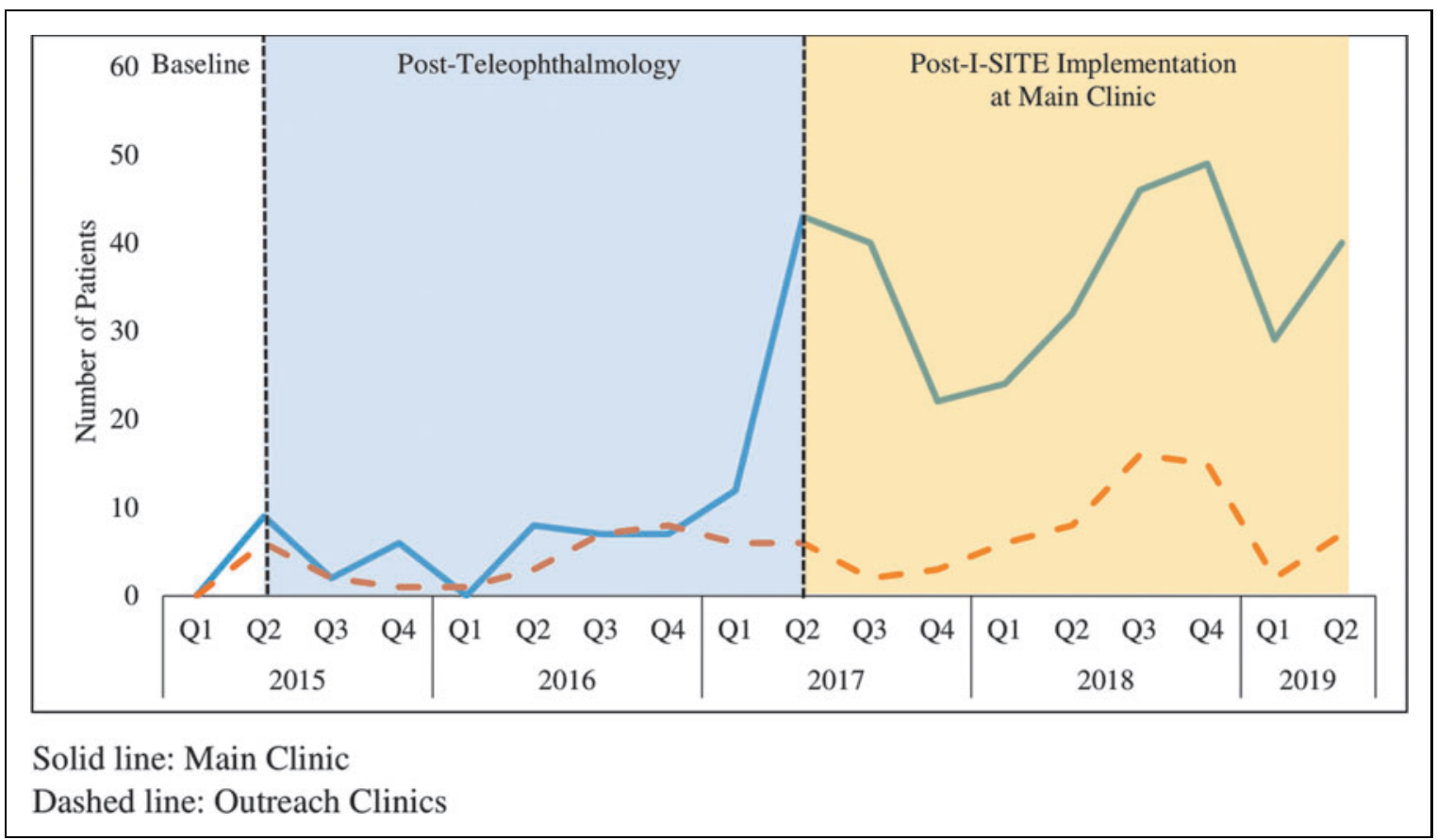

Fig. 1. Teleophthalmology use at Mile Bluff Main and Outreach clinics by quarter.

within a health system. All research activities were conducted in accordance with the Declaration of Helsinki and all federal and state laws.

\section{Results}

\section{TELEOPHTHALMOLOGY USE}

Teleophthalmology use increased over fourfold after initial I-SITE implementation at the Main clinic, between quarter 2 of 2016 and quarter 2 of 2017 (Fig. 1). The increase in teleophthalmology use was sustained 2 years after initial I-SITE implementation through quarter 2 of 2019. Mile Bluff patients had an overall 5.73 increased odds $(p<0.001)$ of using teleophthalmology post-I-SITE implementation compared with post-teleophthalmology. The magnitude of this increased odds of using teleophthalmology was much greater at the Main clinic than at the Outreach clinics (odds ratio $[\mathrm{OR}]=10.0$ vs. $1.69, p<0.001)$.

\section{DIABETIC EYE SCREENING RATES}

Survey response rates between 2015 and 2019 ranged from $74.8 \%$ to $90.3 \%$ among patients whom we were able to contact and $39.5-60.2 \%$ among all patients whom we attempted to contact (data not shown). In 2018, the survey response rate was 90.3\% (326/361) among patients whom we were able to contact and 51.3\% (326/635) among all patients whom we attempted to contact (e.g., including those that either did not pick up the phone or had an out-of-date or nonworking phone number). Patients whom we were able to contact $(n=361)$ were older ( $p=0.03$ ) and more likely to be women ( $p=0.003$ ), with no difference in ethnicity ( $p=0.41)$, compared with those whom we were unable to contact $(n=274)$.

\section{Table 1. Demographics of Patient Telephone Survey} Participants in $2018(N=326)$

\begin{tabular}{l|c} 
PATIENT CHARACTERISTICS & MEDIAN OR PERCENTAGE \\
\hline Age & 68 years (range: 19-95 years) \\
\hline Female & $54.5 \%$ \\
\hline Ethnicity \\
\hline White, non-Hispanic & $97.6 \%$ \\
\hline American Indian/Alaska Native & $0.9 \%$ \\
\hline Asian & $0.9 \%$ \\
\hline Black & $0.3 \%$ \\
\hline Hispanic & $0.3 \%$ \\
\hline Diagnosis of diabetes mellitus & \\
\hline Type I diabetes & $5.2 \%$ \\
\hline Type II diabetes & $94.8 \%$ \\
\hline Median hemoglobin A1c level & 58 mmol/mol (range: $27-132 \mathrm{mmol} / \mathrm{mol}$ ) \\
\hline
\end{tabular}


Table 2. Diabetic Eye Screening Rates at Mile Bluff Medical Center (Overall, Main, and Outreach Clinics) and National Averages by Year ${ }^{4}$

POST-I-SITE

POSTIMPLEMENTATION TIME PERIOD BASELINE TELEOPHTHALMOLOGY

\begin{tabular}{l|c|c|c|c|c}
\hline Year & 2015 & 2016 & 2017 & 2018 & 2019 \\
\hline Overall & $47.4 \%$ & $60.7 \%$ & $58.4 \%$ & $60.2 \%$ & $64.1 \%$ \\
\hline Main clinic & $49.0 \%$ & $61.0 \%$ & $61.5 \%$ & $60.2 \%$ & $67.4 \%$ \\
\hline Outreach clinics & $44.0 \%$ & $60.1 \%$ & $54.4 \%$ & $60.2 \%$ & $59.2 \%$ \\
\hline National average & $53.7 \%$ & $53.6 \%$ & $55.0 \%$ & $55.9 \%$ & $\begin{array}{l}\text { Data not } \\
\text { available }\end{array}$ \\
\hline
\end{tabular}

Survey participants $(n=326)$ were predominately white, non-Hispanic with a median age of 68 years (Table 1). There were no significant demographic differences in age, gender, or ethnicity between survey participants $(n=326)$ versus nonparticipants $(n=35)$, or between survey participants from the Main $(n=88)$ versus Outreach clinics $(n=238)$.

Overall diabetic eye screening rates at Mile Bluff increased from $47.4 \%$ (below the national average) in 2015 to above the national average at $60.7 \%$ and $58.4 \%$ post-teleophthalmology in 2016 and 2017, respectively (Table 2). Screening rates further increased to $60.2 \%$ and $64.1 \%$ at 1 and 2 years post-I-SITE implementation in 2018 and 2019, respectively. Similarly, diabetic eye screening rates at the Main and Outreach clinics, respectively, increased from $49.0 \%$ and $44.0 \%$ at baseline to $61.0-61.5 \%$ and $54.4-60.1 \%$ postteleophthalmology (Table 2). Those increases in screening rates were sustained at $60.2-67.4 \%$ and 59.2-60.2\% post-I-SITE implementation (Table 2 and Fig. 2).

Overall, the odds of a patient receiving any diabetic eye screening increased significantly compared with baseline both post-teleophthalmology $(\mathrm{OR}=1.75, p<0.001)$ and post-I-SITE implementation $(\mathrm{OR}=2.0, p<0.001)$. However, there was no significant difference between Main versus Outreach clinics ( $\mathrm{OR}=1.85$ vs. $2.27, p=0.07)$ when comparing the increase in diabetic eye screening between baseline and post-I-SITE implementation. The sustained increase in screening rates at the Outreach clinics was likely because of spillover effects from I-SITE implementation at the Main Clinic and was mainly driven by increased use of in-person dilated eye examinations for diabetic eye screening.

The likelihood of obtaining any form of diabetic eye screening increased with age (OR $=1.02$ per year of age, 95\%

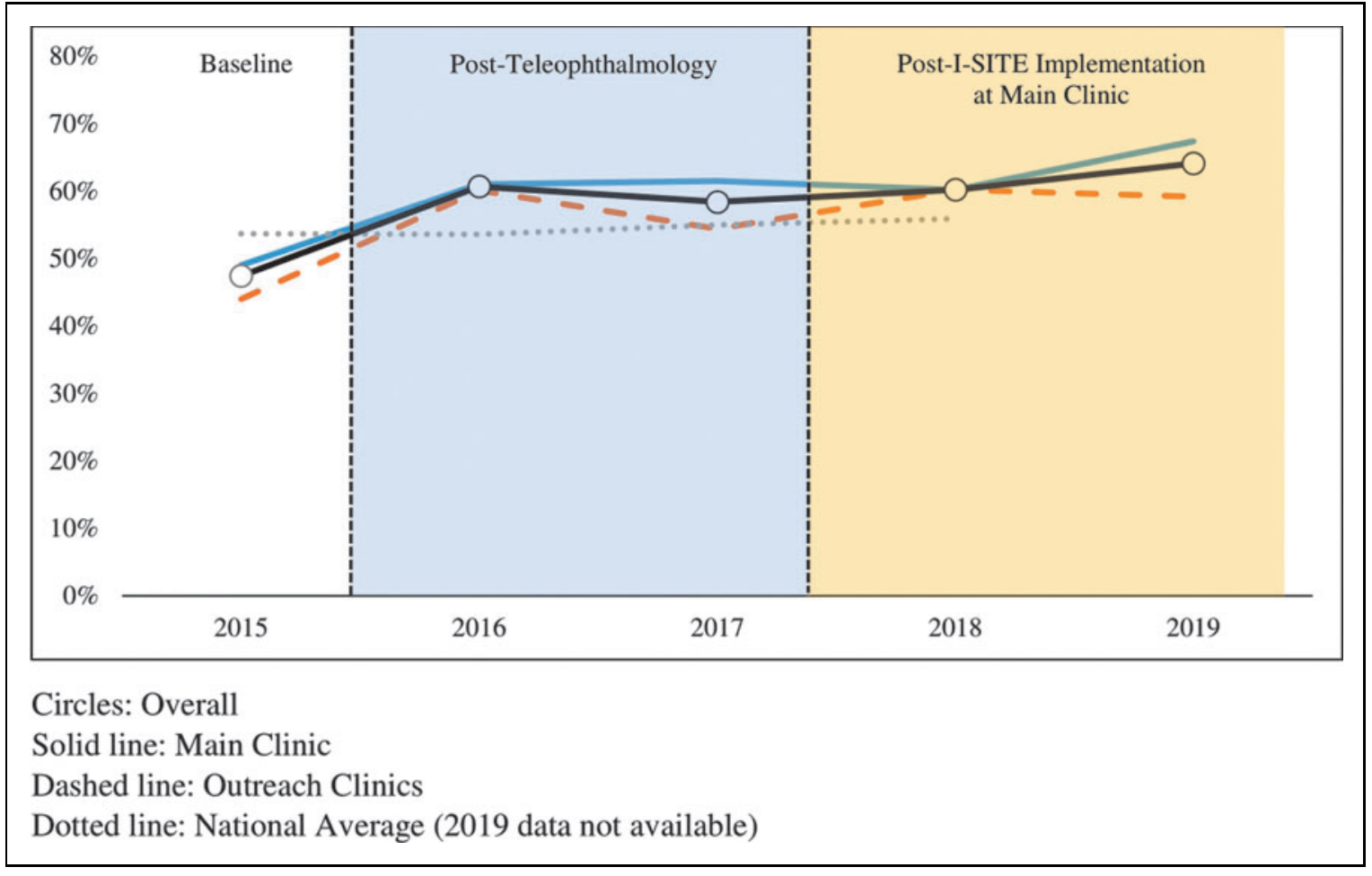

Fig. 2. Diabetic eye screening rates at Mile Bluff overall, Main clinic, Outreach clinics, and national averages by year. ${ }^{4}$ 


\section{LIU ET AL.}

confidence interval $[\mathrm{CI}]=1.01-1.03, p<0.001$ ), but was not associated with gender $(p=0.36)$ or hemoglobin A1c $(p=0.79)$.

\section{USE OF TELEOPHTHALMOLOGY VERSUS IN-PERSON DILATED EYE EXAMINATIONS FOR DIABETIC EYE SCREENING}

Among patients adherent with diabetic eye screening in our 2018 survey, the majority had in-person dilated eye examinations (86.8\%) rather than teleophthalmology (13.2\%). No patients underwent both types of screening in the preceding 12 months. Patients were more likely to use teleophthalmology for diabetic eye screening (rather than an in-person dilated eye examination) if they were younger $(\mathrm{OR}=0.98$ per year of age, 95\% CI =0.96-0.99, $p=0.02)$, male (OR $=1.98$, 95\% CI $=1.28-$ 3.06, $p=0.002$ ), or had a PCP at the Main clinic (OR=4.88, 95\% $\mathrm{CI}=2.31-10.3, p<0.001)$. There was no association between hemoglobin A1c level and the likelihood of utilizing teleophthalmology ( $p=0.18$ ) versus an in-person dilated eye examination.

\section{Discussion}

Our stakeholder-based implementation program achieved a significant increase in overall teleophthalmology use and maintained increased post-teleophthalmology diabetic eye screening rates when implemented within a multipayer U.S. primary care clinic. Overall diabetic eye screening significantly increased from baseline to post-teleophthalmology, as well as from baseline to post-I-SITE implementation.

This increase in overall diabetic eye screening rates moves Mile Bluff from performing below the national average into the top quartile of health systems nationally on this key quality measure. ${ }^{4}$ It also meets the diabetic eye screening rate criterion (60\% or greater) for achieving National Committee for Quality Assurance (NCQA) Diabetes Recognition Program status. ${ }^{25}$ Of note, these increases in teleophthalmology use and screening rates resulted from I-SITE implementation at only one of the five primary clinics in this health system.

In contrast to earlier studies, I-SITE maintained postteleophthalmology diabetic eye screening rates without relying upon either government ${ }^{13}$ or research funding ${ }^{11,14}$ for its clinical operation. In a randomized clinical trial, Mansberger et al. ${ }^{11}$ found that despite a promising initial increase, screening rates in the teleophthalmology group declined rapidly by 18 months and subsequently ranged between $40 \%$ and 55\% even when telemedicine also was offered at no cost to patients in the control group. Our study had an additional barrier in that patients were clinically billed. In addition, Mamillapalli et al. ${ }^{26}$ found only a modest increase in screening rates from
$56.5 \%$ to $59.3 \%$ using teleophthalmology in an endocrinology clinic. This suggests that higher baseline screening rates may make it more difficult, rather than easier, to achieve an improvement because patients who are more willing to obtain screening are already doing so and fewer patients are eligible for teleophthalmology.

Of interest, we found no significant difference between the Main and Outreach clinics in the increased likelihood of patients obtaining diabetic eye screening post-I-SITE implementation compared with baseline. Main clinic patients who previously obtained in-person dilated eye examinations may have substituted these with teleophthalmology, which has been described by Mansberger et al. ${ }^{11}$ Furthermore, there was likely a spillover effect ${ }^{27}$ from I-SITE implementation at the Main clinic upon the Outreach clinics. Staff from all clinics were present at regularly scheduled staff meetings where data were shared on teleophthalmology use and screening rates, and some providers practiced at both Main and Outreach clinics. Thus, Outreach clinic providers increased their use of in-person dilated eye examinations for diabetic eye screening because of exposure to some of the implementation strategies tested in the Main clinic.

Our study agreed with earlier work showing that younger patients and men were more likely to utilize teleophthalmology than in-person dilated eye examinations for diabetic eye screening, ${ }^{26}$ and that hemoglobin A1c level was not associated with either teleophthalmology use or diabetic eye screening adherence. ${ }^{28}$ Older patients are more likely to have coexisting ocular conditions ${ }^{29}$ (cataract, glaucoma, etc.) that require inperson visits to an eye care provider who concurrently performs their in-person dilated eye examination for diabetic eye screening.

Limitations of our study include that it was conducted in a single U.S. health system and that the patient population was predominately white and non-Hispanic, reflecting the population of rural Wisconsin. We did not assess the effect of insurance status because the vast majority of our patients were insured. The proportion of patients with type 2 diabetes (95\%) in our study is consistent with U.S. and worldwide averages. ${ }^{30,31}$ If there had been a greater proportion of patients with type 1 diabetes, we would expect lower screening rates at baseline (which could have impacted the screening rates achieved in our study). This is because the average age of onset for type 1 diabetes is earlier than for type 2 diabetes and younger adults are less likely to obtain diabetic eye screening. ${ }^{28,30}$

We defined the adherence window for diabetic eye screening as within the preceding 12 months based on AAO guidelines. ${ }^{24}$ However, it is becoming increasingly accepted to extend the diabetic eye screening interval from 12 to 24 months for 
patients with a history of mild or no retinopathy per ADA guidelines. ${ }^{2}$ We did not use the ADA guidelines in our study because we did not have access to historical data on retinopathy severity from all patients. Thus, by using the more stringent AAO guidelines, we may have underestimated the proportion of patients' adherence with diabetic eye screening.

In conclusion, our stakeholder-based teleophthalmology implementation program increased teleophthalmology use and maintained post-teleophthalmology increases in diabetic eye screening rates in a multipayer U.S. health system. To date, I-SITE is the only freely available teleophthalmology implementation program specifically designed for U.S. health systems outside the VA Health System. Stakeholder-based implementation may increase the long-term reach and effectiveness of teleophthalmology to reduce vision loss from diabetes. Our approach may improve integration of telehealth interventions into primary care.

\section{Acknowledgments}

The authors acknowledge Kyle W. McDaniel, MPH and Mark Banghart, MS for their assistance with this article. The authors also acknowledge the Wisconsin Network for Research Support (WINRS), Wisconsin Research and Education Network (WREN), and the Primary Care Academics Transforming Healthcare (PATH) Writing Collaborative for their feedback on stakeholder recruitment methodology and the development of implementation program materials. The authors thank the Mile Bluff Medical Center Diabetes Patient Advisory Council and Diabetes Quality Care Team for providing feedback to refine our research results.

\section{Authors' Contributions}

Y.L., J.E.M., M.P., and M.A.S. conceptualized and designed the study. Y.L., T.D.M., J.E.M., and M.A.S. contributed to developing and testing the implementation program. Y.L., J.N.C., N.J.Z., and D.B. conducted the stakeholder meetings. Y.L., J.N.C., A.T.D., N.J.Z., and M.P. gathered and analyzed the data. J.E.M., L.J.L., M.P., T.D.M., N.J.Z., D.B., T.D.B., and M.A.S. contributed to the discussion and reviewed/edited the article. Y.L., J.N.C., A.T.D., and L.J.L. wrote the article. Y.L. accepts full responsibility for the data, analyses, interpretation, conduct of the study, decision to publish, and had full access to all the data.

\section{Disclosure Statement}

No competing financial interests exist.

\section{Funding Information}

This work was supported by NIH/NEI K23 EY026518, NIH/ NEI P30 EY016665, and a Wisconsin Partnership Program New Investigator Award. It was also supported, in part, by an institutional grant from Research to Prevent Blindness, New York City, New York, USA to the University of Wisconsin School of Medicine and Public Health, Department of Ophthalmology and Visual Sciences. The funders had no influence on the design or results of the study.

\section{REFERENCES}

1. Centers for Disease Control and Prevention. National diabetes statistics report: Estimates of diabetes and its burden in the United States, 2014. Available at https://stacks.cdc.gov/view/cdc/23442/cdc_23442_DS1.pdf? (last accessed on May 26, 2020).

2. American Diabetes Association. 11. Microvascular complications and foot care: Standards of medical care in diabetes-2020. Diabetes Care 2020:43:S135-S151.

3. Lynch MG, Maa AY. Diagnostic time for teleophthalmic care. JAMA Ophthalmol 2019;137:808-809.

4. National Committee for Quality Assurance. Comprehensive Diabetes Care (CDC). Available at https://www.ncqa.org/hedis/measures/comprehensivediabetes-care/ (last accessed on April 2, 2020).

5. Scanlon PH. The English national screening programme for sight-threatening diabetic retinopathy. J Med Screen 2008;15:1-4.

6. Liew $G$, Michaelides $M$, Bunce $C$. A comparison of the causes of blindness certifications in England and Wales in working age adults (16-64 years), 19992000 with 2009-2010. BMJ Open 2014;4:6.

7. Gibson DM. Estimates of the percentage of US adults with diabetes who could be screened for diabetic retinopathy in primary care settings. JAMA Ophthalmol 2019;137:440-444.

8. Silva PS, Aiello LP. Telemedicine and eye examinations for diabetic retinopathy: A time to maximize real-world outcomes. JAMA Ophthalmol 2015;133:525526.

9. Zimmer-Galler IE, Kimura AE, Gupta S. Diabetic retinopathy screening and the use of telemedicine. Curr Opin Ophthalmol 2015;26:167-172.

10. Centers for Medicare and Medicaid Services. List of telehealth services. Available at https://www.cms.gov/Medicare/Medicare-General-Information/ Telehealth/Telehealth-Codes (last accessed on May 26, 2020).

11. Mansberger SL, Sheppler C, Barker G, Gardiner SK, Demirel S, Wooten K, Becker TM. Long-term comparative effectiveness of telemedicine in providing diabetic retinopathy screening examinations a randomized clinical trial. JAMA Ophthalmol 2015;133:518-525.

12. Modjtahedi BS, Theophanous C, Chiu S, Luong TQ, Nguyen N, Fong DS. Twoyear incidence of retinal intervention in patients with minimal or no diabetic retinopathy on telemedicine screening. JAMA Ophthalmol 2019;137:445-448.

13. Daskivich $L P$, Vasquez $C$, Martinez $C$, Jr., Tseng $C H$, Mangione CM. Implementation and evaluation of a large-scale teleretinal diabetic retinopathy screening program in the Los Angeles County Department of Health Services. JAMA Intern Med 2017;177:642-649.

14. Jani PD, Forbes L, Choudhury A, Preisser JS, Viera AJ, Garg S. Evaluation of diabetic retinal screening and factors for ophthalmology referral in a telemedicine network. JAMA Ophthalmol 2017;135:706-714.

15. Hussey $P$, Anderson GF. A comparison of single- and multi-payer health insurance systems and options for reform. Health Policy 2003;66:215-228.

16. Liu Y, Zupan NJ, Swearingen R, Jacobson N, Carlson JN, Mahoney JE, Klein R, Bjelland TD, et al. Identification of barriers, facilitators and system-based 


\section{LIU ET AL.}

implementation strategies to increase teleophthalmology use for diabetic eye screening in a rural US primary care clinic: A qualitative study. BMJ Open 2019;9:e22594.

17. Bouskill K, Smith-Morris C, Bresnick G, Cuadros J, Pedersen ER. Blind spots in telemedicine: A qualitative study of staff workarounds to resolve gaps in diabetes management. BMC Health Serv Res 2018;18:617.

18. Centers for Healthcare Enhancement Systems Studies. What is NIATx? Available at https://www.niatx.net/what-is-niatx/ (last accessed on June 30, 2020).

19. County Health Ranking Roadmaps. Juneau County Demographics. Available at https://www.countyhealthrankings.org/app/wisconsin/2019/rankings/juneau/ county/outcomes/overall/snapshot (last accessed on February 28, 2020).

20. American Telemedicine Association. Telehealth practice recommendations for diabetic retinopathy, second edition. Telemed J E Health 2011;17:814-837.

21. Perry CK, Damschroder $\sqcup$, Hemler JR, Woodson TT, Ono SS, Cohen DJ. Specifying and comparing implementation strategies across seven large implementation interventions: A practical application of theory. Implement Sci 2019;14:32.

22. Holley CD, Lee PP. Primary care provider views of the current referral-toeye-care process: Focus group results. Invest Ophthalmol Vis Sci 2010;51: 1866-1872.

23. Fowles JB, Rosheim K, Fowler EJ, Craft C, Arrichiello L. The validity of self-reported diabetes quality of care measures. Int J Qual Health Care 1999;11:407-412.

24. Flaxel CJ, Bailey ST, Fawzi A, Lim Jl, Adelman RA, Vemulakonda A, Ying G-S. Diabetic retinopathy Preferred Practice Pattern 2019. Available at https:// www.aao.org/preferred-practice-pattern/diabetic-retinopathy-ppp (last accessed on April 2, 2020).

25. National Committee for Quality Assurance. 2015 Diabetes Recognition Program (DRP) requirements. Available at https://recognitionportal.ncqa.org/ documents/2015\%20DRP\%20Requirements.pdf (last accessed on May 26, 2020).

26. Mamillapalli CK, Prentice JR, Garg AK, Hampsey SL, Bhandari R. Implementation and challenges unique to teleretinal diabetic retinal screening
(TDRS) in a private practice setting in the United States. J Clin Trans/ Endocrinol 2020;19:100214.

27. Ford I, Norrie J. Pragmatic trials. N Engl J Med 2016;375:454-463.

28. Eppley SE, Mansberger SL, Ramanathan S, Lowry EA. Characteristics associated with adherence to annual dilated eye examinations among US patients with diagnosed diabetes. Ophthalmology 2019;126:1492-1499.

29. Vision and Aging Resources. Available at https://www.nei.nih.gov/learn-abouteye-health/resources-for-health-educators/vision-and-aging-resources (last accessed on March 9, 2020).

30. Xu G, Liu B, Sun Y, Du Y, Snetselaar LG, Hu FB, Bao W. Prevalence of diagnosed type 1 and type 2 diabetes among US adults in 2016 and 2017: Population based study. BMJ 2018;362:k1497.

31. National Diabetes Statistics Report, 2020. Atlanta, GA: Centers for Disease Control and Prevention, 2020.

Address correspondence to:

Yao Liu, MD, MS

Department of Ophthalmology and Visual Sciences

University of Wisconsin School of Medicine and Public Health

2870 University Avenue, Suite 206

Madison, WI 53705

USA

E-mail:yaoliumd@gmail.com

Received: July 7, 2020

Revised: September 23, 2020

Accepted: September 29, 2020

Online Publication Date: November 19, 2020 